\title{
Residência Integrada em Saúde do Grupo Hospitalar Conceição: uma Proposta de Formação de Cirurgiões-Dentistas em Saúde da Família e Comunidade.
}

Health Integrated Residency of Conceição Hospital Group: a Proposal for Dentists Training in Community and Family Health.

Camila Samara Funk ${ }^{1}$, Daniel Demétrio Faustino-Silva ${ }^{2}$, Etiane Malacarne ${ }^{3}$, Raquel Porciúncula Rodrigues ${ }^{3}$, Rossana Rad Fernandez ${ }^{3}$

\section{Abstract}

The Residencies in Health, mode of lato sensu post-graduation, appears as a strategy for human resource training for the Brazilian Public Health System (SUS) with profile to be able to change the current practices from a new culture of intervention and understanding in health. This trial seeks to present the proposal of the Conceição Hospital Group Integrated Health Residency (RIS/GHC) to the Dentistry group, emphasis in Community and Family Health. The program consists itself on the teaching-research-assistance and objectives to training qualified dentists to the needs of the SUS, able to work in interdisciplinary teams, from care to management, beyond to providing subsidies for the research development, in a practice based in conceptions of humanization and health surveillance, taking the principles of Primary Health Care as guiding axes. The teachinglearning process is developed in two years, consisting of theoretical and practical field activities - set of theories and practices common to several professions, and core - set of knowledge and practices specific to each occupation. The theoretical activities account about $20 \%$ of the total workload, developed through research activities, case studies, workshops and seminars, interspersed with practice in the Community Health Service Unities and in the stages scheduled in the emphasis and core programs. This post-graduation mode intends to minimize the historical deficiencies of assistance and training in the field of dentistry, forming political, critical and active citizens, to seek the collective construction of a health project fair and qualified.

Keywords: Community Dentistry, Dental Staff, Single Health System.

\section{Resumo}

As Residências em Saúde, modalidade de pós-graduação lato sensu, configuram-se como uma estratégia de formação de recursos humanos para o Sistema Único de Saúde (SUS) com perfil capaz de modificar as práticas atuais a partir de uma nova cultura de intervenção e entendimento da saúde. Este ensaio busca apresentar a proposta da Residência Integrada em Saúde do Grupo Hospitalar Conceição (RIS/GHC) para o núcleo da Odontologia, ênfase Saúde da Família e Comunidade. O programa constitui-se no tripé ensinoassistência-pesquisa e objetiva formar cirurgiões-dentistas qualificados às exigências do SUS, capazes de trabalhar em equipe interdisciplinar, da atenção à gestão, além de fornecer subsídios para o desenvolvimento de pesquisas, numa prática alicerçada nas concepções de humanização e vigilância em saúde, tendo os princípios da Atenção Primária à Saúde como eixos orientadores. $\mathrm{O}$ processo de ensino-aprendizagem em serviço é desenvolvido em dois anos, constituídos por atividades teóricas e práticas de campo conjunto de saberes e práticas comuns a várias profissões, e núcleo conjunto de saberes e práticas específicos a cada profissão. As atividades teóricas correspondem a cerca de $20 \%$ do total da carga horária, desenvolvidas na forma de atividades de pesquisa, estudos
${ }^{1}$ Odontóloga e preceptora do Programa de Residência Integrada em Saúde - RIS Ênfase Saúde da Família e Comunidade do Serviço de Saúde Comunitária - SSC do Grupo Hospitalar Conceição - GHC. Mestre em Saúde Coletiva - UFRGS.

${ }^{2}$ Odontólogo e preceptor do Programa de Residência Integrada em Saúde - RIS Ênfase Saúde da Família e Comunidade do Serviço de Saúde Comunitária - SSC do Grupo Hospitalar Conceição-GHC. Especialista em Saúde Coletiva e Mestre em Clínica Odontológica - Odontopediatria UFRGS.

${ }^{3}$ Odontólogas Residentes do programa de Residência Integrada em Saúde - RIS Ênfase Saúde da Família e Comunidade do Grupo Hospitalar Conceição-GHC.

Correspondência: Camila Samara Funk

Endereço: Rua Cananéia, 220, Vila Jardim - CEP 91330-580, Porto Alegre RS, Brasil

E-mail: camilafunk@hotmail.com

Data de Submissão: 06/02/2011

Data de Aceite: $07 / 11 / 2011$

de caso, oficinas e seminários, intercalados com a prática nas Unidades da Saúde Comunitária e nos estágios previstos no programa de ênfase e núcleo. Esta modalidade de pós-graduação pretende dirimir as deficiências históricas de assistência e formação na área da Odontologia, formando cidadãos políticos, críticos e ativos, que busquem a construção coletiva de um projeto de saúde justo e qualificado.

Palavras-chaves: Odontologia Comunitária, Recursos Humanos em Odontologia, Sistema Único de Saúde.

\section{A Formação de Cirurgiões-Dentistas para o SUS: 0 Papel das Residências Multiprofissionais}

A necessidade de produzir mudanças efetivas nas práticas de atenção à saúde no Brasil, compatíveis com os novos princípios e diretrizes do Sistema Único de Saúde (SUS), mobiliza constantemente o debate e a construção de estratégias que gerem esse enfrentamento, incluindo as políticas de formação de recursos humanos, tanto em nível técnico, como de graduação e pósgraduação. Esse novo modo de pensar/agir em saúde determina a quebra de referências históricas e culturais importantes, baseadas no modelo biomédico assistencial privatista, impactando especialmente os núcleos profissionais ditos "duros", como a Odontologia, com formação ainda fortemente vinculada as questões biológicas, a tecnologia dura de equipamentos e materiais e a especialização/compartimentação do saber.

A reforma curricular desenvolvida em muitos cursos de graduação tensiona a mudança, mas ainda é recente para uma análise efetiva do impacto nas práticas profissionais, nas condições de saúde bucal da população e no próprio mercado de trabalho acessível a este novo perfil profissional. No âmbito da pós-graduação, as Residências Integradas em Saúde, na modalidade de lato sensu, 
configuram-se como uma importante estratégia de transformação, buscando formar profissionais com perfil capaz de modificar as práticas atuais, a partir de uma nova cultura de intervenção e entendimento da saúde.

Nesse sentido, o presente ensaio tem como objetivo apresentar a experiência da Residência Integrada em Saúde (RIS) do Grupo Hospitalar Conceição (GHC) na formação de cirurgiõesdentistas na ênfase Saúde da Família e Comunidade e discutir o papel desse profissional na Atenção Primária à Saúde (APS) no contexto do SUS.

\section{Residência Integrada em Saúde da Família e Comunidade do GHC: uma Construção Histórica}

As Residências em Saúde Comunitária como modalidade de pós-graduação iniciam-se no Brasil no ano de 1976, no Estado do RS, por iniciativa da Secretaria Estadual de Saúde (SES-RS). A primeira turma, alocada na Unidade Sanitária São José do Murialdo, foi direcionada ao núcleo médico, para formação em Medicina Geral Comunitária. Sua proposta incluía formar profissionais com uma visão integrada entre saúde clínica, saúde mental e saúde pública, com perfil humanista e crítico e com competência para uma boa resolubilidade das necessidades de saúde da comunidade (BRASIL, 2006).

No ano de 1978 é iniciada a Residência Multiprofissional em Saúde Comunitária, também na Unidade Sanitária Murialdo, incorporando enfermeiros, psicólogos, assistentes sociais, nutricionistas e médicos veterinários, sendo a inclusão de cirurgiõesdentistas no ano de 1980 (MOYSÉS et al., 2008). Esta nova proposta de formação surge durante o movimento da Reforma Sanitária, no auge da ditadura militar, em resistência ao modelo biologicista, centrado na doença e estruturado na medicalização dos sujeitos e na especialização e fragmentação do conhecimento (BRASIL, 2006).

Neste cenário e por influência deste, cria-se, em 1985, no Grupo Hospitalar Conceição (GHC) de Porto Alegre, o Serviço de Medicina de Família, com a instalação de uma Unidade Básica de Saúde nas dependências de um dos hospitais do grupo - o Hospital Nossa Senhora Conceição (HNSC). A instalação desta Unidade tinha dois objetivos: atender às necessidades de saúde dos moradores da área próxima ao hospital e adequar-se às exigências da Comissão Nacional de Residência Médica para o credenciamento do Programa de Residência em Medicina Geral Comunitária. Entre 1985 e 1995 foram abertas mais 11 unidades de saúde, originando o chamado Serviço de Saúde Comunitária (SSC). A criação dessa nomenclatura, em 1989, reflete uma mudança no conceito do serviço, que deixa de ser restrito em Medicina de Família e começa a incorporar profissionais de outras categorias, buscando estratégias que respondam mais amplamente as necessidades de saúde das comunidades, famílias e indivíduos atendidos nas unidades (MOYSÉS et al., 2008).

$\mathrm{Na}$ premência de uma reordenação do modelo de APS no Brasil, em 1994, o Ministério da Saúde (MS) lançou o então denominado Programa de Saúde da Família (PSF), posteriormente denominado Estratégia de Saúde da Família (ESF), e buscou no Serviço de Saúde Comunitária do GHC apoio técnico para sua implantação, pois há 11 anos esse serviço trabalhava com ênfase na APS (MOYSÉS et al., 2008).

Orientado por este novo modelo de atenção e a necessária formação de recursos humanos para sua implementação e efetivação, em 1999 o Departamento de Atenção Básica (DAB) da Secretaria de Atenção à Saúde do MS, junto a atores do Movimento Sanitário, articularam-se na proposta de criar, reavivar e reinventar as
Residências em Saúde da Família. O objetivo era criar um modelo de Residência Multiprofissional, onde embora fossem preservadas as especialidades de cada profissão envolvida, seria criada uma área comum, especialmente vinculada ao pensamento da tradicional saúde pública, acrescida de valores como a promoção da saúde, a integralidade da atenção e o acolhimento. Assim, em 2002 foram criadas 19 residências Multiprofissionais em Saúde da Família financiadas pelo MS, com formatos diversificados, mas dentro da perspectiva de trabalhar integradamente com todas as profissões da saúde (BRASIL, 2006).

Em 2004, por meio de um projeto também financiado pelo MS, é implantada no GHC a Residência Integrada em Saúde (RIS), inicialmente nas ênfase em Saúde da Família e Comunidade (SFC), Saúde Mental (SM) e Terapia Intensiva (TI). O SSC passa a ser o espaço de formação da ênfase SFC, com residentes de várias profissões da área da saúde, incluindo a Odontologia. Inicialmente o número de vagas para Odontologia foi de 5 por ano. Devido a grande procura nos processos seletivos e a importância dessa área para a Estratégia de Saúde da Família (ESF), as vagas têm sido aumentadas gradativamente, sendo que atualmente são em número de 9 por ano.

A Residência em Área Profissional da Saúde e também a Comissão Nacional de Residência Multiprofissional em Saúde (CNRMS) foram criadas pela Lei $n^{\circ} 11.129 / 2005$, iniciando o processo de regulamentação dos Programas. A Residência em Área Profissional da Saúde fica definida como modalidade de ensino de pós-graduação lato sensu e têm como objetivo "favorecer a inserção qualificada dos/as jovens profissionais da saúde no mercado de trabalho, particularmente em áreas prioritárias do SUS", oferecendo bolsas através de projetos aprovados pelo MS para as profissões definidas por ele como "da área da saúde", excetuada a médica que apresenta programa específico (BRASIL, 2005a). As normas para a implementação e execução do Programa de Bolsas foi fixada pela portaria 1.111/GM/MS/2005 (BRASIL, 2005b). Também em 2005, através da Portaria Interministerial $n^{\circ} 2.117$ foi formalizada a ação articulada entre o Ministério da Educação (MEC) e o MS para a formação e o desenvolvimento de recursos humanos na saúde, formalizando a aproximação entre as políticas de educação e saúde esboçadas na Constituição Federal de 1988 (BRASIL, 2005c).

A Portaria Interministerial $n^{\circ} 45 / 2007$ determinou que os Programas de Residência Multiprofissionais em Saúde (PRMS) e Residência em Área Profissional da Saúde devem ser orientados pelos princípios e diretrizes do SUS, a partir das necessidades e realidades locais e regionais, de forma a contemplar os seguintes eixos norteadores: cenários de educação em serviço; concepção ampliada de saúde; política nacional de educação e desenvolvimento no SUS; estratégias pedagógicas capazes de garantir a formação integral e interdisciplinar e que considerem os atores envolvidos como sujeitos do processo de ensino-aprendizagem-trabalho e protagonistas sociais; integração ensino-serviço-comunidade; integração de saberes e práticas que permitam a consolidação do processo de formação em equipe; integração com diferentes níveis de formação na área da saúde; articulação da Residência Multiprofissional e em Área Profissional da Saúde com a Residência Médica; descentralização e regionalização contemplando as necessidades locais, regionais e nacionais de saúde; monitoramento e avaliação pactuados para garantir que o sistema de avaliação formativa seja dialógico e abranja a participação dos sujeitos envolvidos; integralidade que contemple todos os níveis da Atenção à Saúde e de Gestão do Sistema (BRASIL, 2007). As regulamentações mais recentes a respeito da RMS são a Resolução $n^{\circ} 2$ e $n^{\circ} 3$, de maio de 2010, que dispõem sobre a organização, o funcionamento e as atribuições da Comissão de Residência Multiprofissional (COREMU) e a período (mínimo de 2 anos), a carga horária (mínimo 
de 5760 horas), avaliação e freqüência dos programas (BRASIL, 2010b).

\section{Os Espaços de Formação da RIS/GHC: um Olhar sobre Campo e Núcleo da Odontologia em Saúde da Família e Comunidade}

A RIS/GHC está estruturada a partir do tripé ensinoassistência-pesquisa e tem o objetivo de especializar profissionais de diversas áreas da saúde para a atuação e o trabalho em equipe, segundo os princípios do SUS (BRASIL, 2009a). A proposta visa, além da formação de um profissional qualificado às exigências do SUS, a formação de um cidadão crítico que busque, em seus espaços de atuação profissional, social e política, a possibilidade de construir, coletivamente, soluções aos problemas que acometem tanto os usuários como o próprio sistema e os trabalhadores da saúde. A formação em serviço, desenvolvida sob a ótica da interdisciplinaridade e da humanização da atenção, visa a propiciar uma melhor atenção à saúde dos usuários, além de melhorar a qualidade de vida de todos - dos usuários dos serviços, que serão vistos em sua integralidade; dos profissionais em formação, ao aumentarem sua capacidade de diálogo e alcançarem uma compreensão ampliada da realidade; e dos preceptores e orientadores, pela possibilidade educativo-participativa do trabalho em saúde (BRASIL, 2010b).

O processo de ensino e aprendizagem em serviço é desenvolvido ao longo de dois anos, sendo constituído por atividades de reflexão teórica com todo o grupo de residentes, por atividades teóricas específicas de campo e núcleo em cada área de ênfase e por atividades em serviço (Figura 1). Campo é o conjunto de saberes e práticas comuns a várias profissões ou especialidades, e núcleo é o conjunto de saberes e práticas específicos a cada profissão ou especialidade e marca a diferença entre os membros de uma equipe, com elementos de singularidade, conhecimentos e ações de exclusiva competência de cada profissão ou especialidade (BRASIL, 2006).

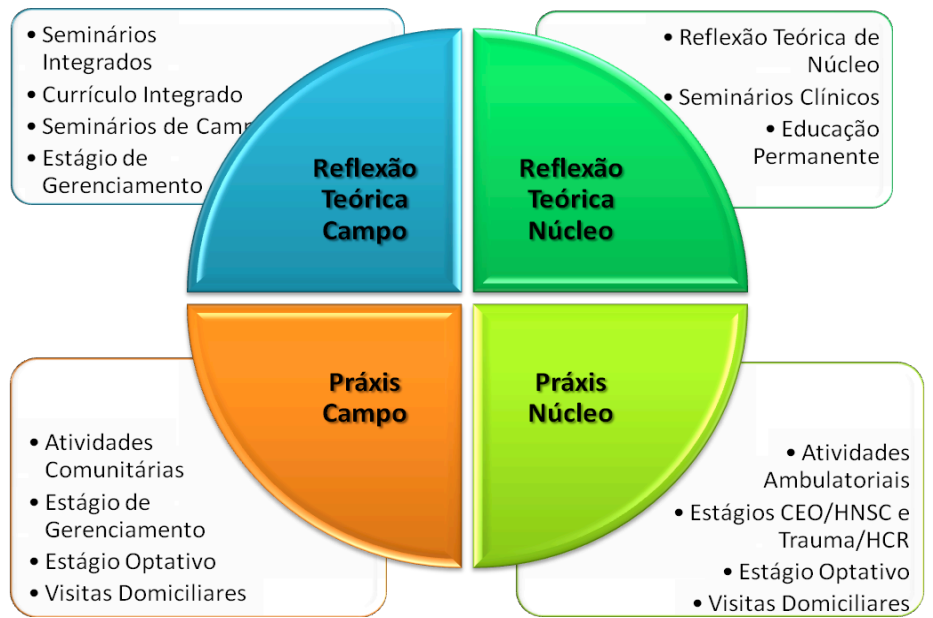

Figura 1) Estrutura base de formação do Núcleo de Odontologia Ênfase Saúde da Família e Comunidade.

As atividades teóricas da RIS/GHC correspondem a cerca de $20 \%$ do total de carga horária, sendo desenvolvidas na forma de atividades de pesquisa, estudos de caso, oficinas e seminários, intercalados com a formação prática em serviço nas Unidades do SSC e outros campos de estágio que correspondem aos outros $80 \%$ (BRASIL, 2010a).

Nas atividades de reflexão teórica integrada participam todos os residentes das quatro ênfases da RIS/GHC, atualmente, SFC, SM, Atenção ao Paciente Crítico e Oncologia-Hematologia, divididos em turmas para residentes de primeiro e segundo anos. Estas são desenvolvidas semanalmente por um período de três horas e abordam diversas temáticas, segundo os eixos: seminários de pesquisa, que visam discutir e subsidiar as propostas de investigações a serem desenvolvidas no decorrer da residência; estudos de caso visam discutir uma situação de saúde do cotidiano, buscando problematizar os vários olhares necessários ao cuidado integral; oficinas temáticas, definidas pelos residentes a partir do estudo de problemas e visam ao aprofundamento teórico e a troca de experiências de trabalho inter-relacionadas e políticas de saúde, visa possibilitar a apropriação e discussão dos princípios e conceitos fundamentais que envolvem a atenção em saúde no SUS, bem como as principais políticas (BRASIL, 2009b).

Os seminários ditos de campo são realizados nos campos de formação, isto é, nas Unidades do SSC e visam integrar residentes de primeiro e segundo anos, preceptores e orientadores locais, aprofundando temas relacionados as atividades de formação em serviço vividas cotidianamente, na perspectiva da construção coletiva de conhecimento em equipe multidisciplinar (BRASIL, 2009b).

A ênfase de SFC conta ainda com uma experiência inovadora como ferramenta metodológica, o "currículo integrado". Esta atividade agrega todos os residentes da RIS/SFC e da Medicina de Família e Comunidade (RMFC). A partir de uma organização em pequenos grupos multiprofissionais são estudados os atributos da APS (acesso, longitudinalidade, integralidade, coordenação) e ações programáticas durante o primeiro ano da Residência. No segundo ano são elaborados projetos de implementação de equipes de Saúde da Família (ESF) em municípios que não as possuem (BRASIL, 2009b).

Dentro das atividades do segundo ano da residência existe ainda o estágio de gerenciamento, que integra o currículo de campo da RMFC e da RIS/SFC. O objetivo desse estágio é propiciar a vivência e participação ativa do cotidiano gerencial da Unidade de Saúde em que estão inseridos, no contexto do SSC, GHC e SUS. Esta experiência prática e a apropriação de ferramentas teóricometodológicas e operacionais trabalhados ao longo do período de estágio deverão desenvolver entre os residentes habilidades e competências para a gestão de serviços e unidades de atenção primária do SUS e, concomitantemente, contribuir no desenvolvimento gerencial das unidades do SSC (BRASIL, 2010b).

Os residentes ainda têm a oportunidade de liberação de 15 dias por ano para atividades científicas como congressos e seminários, além da possibilidade de dois meses de estágio optativo durante o segundo ano de residência, a ser realizado dentro ou fora da instituição, como atividade complementar a sua área de formação (BRASIL, 2009b).

As atividades práticas de formação em serviço são desenvolvidas cotidianamente durante todo o período da residência (24 meses), tanto junto às equipes de saúde nas quais os residentes estão inseridos como nos demais campos de estágios previstos no programa de cada área de ênfase e núcleo. Essas atividades visam a colocar em prática os referenciais orientadores da RIS/GHC, bem como a desenvolver o perfil profissional do egresso. Nas Unidades de Saúde os residentes participam de atividades ambulatoriais, visitas domiciliares, ações programáticas, atividades comunitárias como Conselho Local de Saúde, grupos e atividades conjuntas aos Pontos de Cultura e Saúde. Nos estágios, realizados no segundo ano, os residentes do núcleo de Odontologia passam pelo Centro de 
Especialidades Odontológicas (CEO) do Hospital Nossa Senhora da Conceição (HNSC), nas áreas de Periodontia, Pacientes Especiais, Odontologia Hospitalar, Cirurgia, Estomatologia e Gerenciamento do CEO. No Hospital Cristo Redentor (HCR) desenvolve-se o estágio em Traumatismo Buco Maxilo Facial. O objetivo, além de ampliar a resolutividade das questões clínicas na atenção primária, é possibilitar o conhecimento da rede de serviços disponíveis, de média e alta complexidade.

A atividade de pesquisa também faz parte da formação na RIS, completando a qualificação do residente como um profissional envolvido com a assistência e com a produção científica, visto que é a partir desta que o conhecimento pode ser construído, atualizado e transmitido. Todos os residentes apresentam um "Trabalho de Conclusão de Residência" - TCR, denominação que abarca diferentes possibilidades em termos dos objetivos e estratégias metodológicas, como pré-requisito à conclusão da Residência. As temáticas dos TCRs deverão estar de acordo com a Política de Pesquisa do GHC, através das linhas definidas institucionalmente e voltadas para a APS/SUS (BRASIL, 2009b).

O núcleo de Odontologia desenvolve suas atividades de reflexão teórica semanalmente e separadamente para residentes de primeiro e segundo ano. Os temas discutidos nesses encontros incluem: Política Nacional de Saúde Bucal, Saúde Bucal no Território, Ações Programáticas de Saúde Bucal, Aplicabilidade dos atributos da APS (Primeiro Contato, Longitudinalidade, Integralidade, Coordenação) em Odontologia, Abordagem Familiar, Visita Domiciliar, Vigilância em Saúde Bucal, Indicadores e Sistema de Informação em Saúde Bucal. Existem ainda seminários semanais nas Unidades de Saúde e mensais no SSC para discussões clínicas com todos os profissionais do núcleo de Odontologia.

\section{Projeto Político Pedagógico e as Diretrizes de Formação: o Perfil do Egresso}

O Grupo Hospitalar Conceição, instituição pública, referência na assistência e ensino, percebendo as potencialidade da formação multiprofissional para a concretização de um novo modelo de atenção à saúde, implementa, em 2004, a Residência Integrada em Saúde (RIS/GHC), sendo a Odontologia um dos núcleos profissionais desenvolvidos na ênfase Saúde da Família e Comunidade. O programa, orientado pelo Projeto Político Pedagógico (PPP), define quatro eixos principais na formação - integralidade, trabalho em equipe, humanização e educação permanente - e objetiva formar cirurgiões-dentistas qualificados para a prática da atenção à saúde no contexto da APS/SUS (Figura 2).

A integralidade pressupõe um conceito amplo de saúde, no qual necessidades bio-psico-socias, culturais e subjetivas dos usuários são (re)conhecidas e orientam políticas e programas visando a redução do risco de doenças e outros agravos, além de promover o acesso universal e igualitário às ações e serviços para a promoção, proteção e recuperação da saúde.

A ênfase SFC direciona o residente ao desenvolvimento de uma prática ampliada, considerando a complexidade do processo saúde-doença e a multiplicidade das necessidades dos usuários, propiciando o desenvolvimento de uma prática integral através de sua inserção nas mais variadas atividades da unidade de saúde e demais serviços, tendo a promoção, a prevenção, e o tratamento integrados na prática clínica; e a abordagem focada no indivíduo, sua família e seu contexto. Além das atividades de prevenção e promoção realizadas individualmente, destacam-se as ações em grupos, as visitas domiciliares, ações junto a organizações comunitárias e articulações intersetorias. Também, a experiência do trabalho em equipe multiprofissional permite que o cirurgião-dentista articule ações que extrapolam a saúde bucal e por isso desenvolva um olhar ampliado.

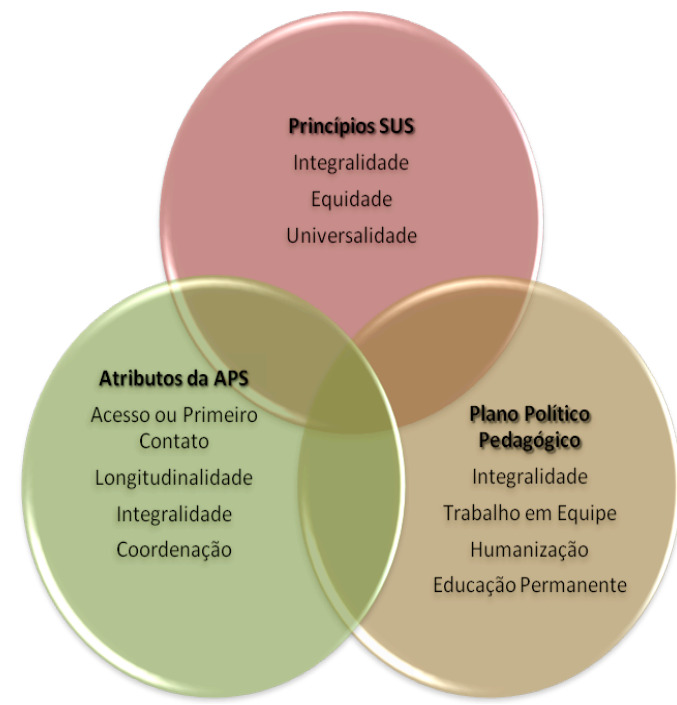

Figura 2. Eixos orientadores da formação.

A integralidade do ponto de vista da saúde bucal em APS engloba ainda outras questões, como a habilidade para resolver aproximadamente $80 \%$ das necessidades de seus usuários. Os demais $20 \%$ das necessidades devem ser contempladas pelos serviços especializados em nível secundário (Centros de Especialidades Odontológicas/CEOs) e terciário (Hospitais) (BRASIL, 2008). É papel da APS estabelecer a coordenação do cuidado entre os níveis de atenção a saúde do usuário, e neste sentido entende-se que os cirurgiões-dentistas egressos da RIS/GHC conheçam, compreendam e dialoguem com a rede de serviços como um todo, ampliando as possibilidades e resolutividade das necessidades percebidas, exercendo seu papel de forma ampla e qualificada.

Já a equidade é experimentada nas vivências em território e em equipe multiprofissional, reconhecendo necessidades, riscos e vulnerabilidades, individuais, familiares e coletivas. Incorporado ao planejamento e demais atividades das equipes, o residente desenvolve a vigilância em saúde, com diferentes dispositivos (áreas de vigilância, ações programáticas, áreas e/ou famílias de risco,...) direcionando ações e facilitando o acesso a indivíduos e grupos de risco.

O trabalho em equipe busca provocar, na interdisciplinaridade, a aproximação dos egressos da multidimensionalidade dos sujeitos e do contexto em que estão inseridos, bem como das formas de expressão de suas demandas, qualificando e ampliando suas ferramentas no campo de atuação. Já a humanização pretende incorporar a dimensão subjetiva do processo, no qual o profissional e o usuário se encontram, se relacionam e experimentam a tomada de decisões conjuntas. A partir de tais subsídios pretende-se que o egresso desenvolva uma prática cotidiana com o conhecimento do ser humano e das relações que o cercam, denotando um compromisso existencial para entender e valorizar o humano. A humanização depende ainda de mudanças das pessoas e do modo de trabalho, significando uma reforma na atenção no sentido de facilitar a construção de vínculos entre equipes e usuários.

O eixo Educação Permanente prevê a formação de um profissional crítico capaz de "aprender a aprender", estruturada a partir da problematizacão do seu processo de trabalho, cujo objetivo é 
a transformação das práticas profissionais e da própria organização do trabalho, tomando como referência as necessidades de saúde dos indivíduos e das populações, da gestão dos trabalhadores em saúde e do controle social.

Complementando e intrinsecamente ligados a esses eixos maiores, a ênfase SFC orienta a formação de cirurgiões-dentistas segundo os atributos da Atenção Primária à Saúde (APS) definidos por Bárbara Starfield (2006) como essenciais: acesso ou primeiro contato, longitudinalidade, integralidade e coordenação; e derivados: orientação familiar e comunitária e competência cultural; e pela prática no território segundo o modelo da vigilância em saúde preconizados pela ESF/SUS.

A reordenação do sistema público de saúde brasileiro prevê o fortalecimento da APS através da ESF visando a aproximação da comunidade, a ampliação do acesso de forma mais equânime e qualificada as reais necessidade de saúde, e a orientação do cuidado aos diferentes níveis de atenção. A APS é capaz de tornar um sistema de saúde mais efetivo, com menores custos, maior satisfação da população e maior equidade, mesmo diante das adversidades sociais. Em vista disso, discute-se incessantemente seus princípios norteadores, nos mais diversos espaços de formação, com a intenção que o cirurgião-dentista egresso tenha $o$ entendimento $e$ aplicabilidade prática destes no sistema de saúde.

A Atenção Primária deve ser considerada a principal porta de entrada (acesso) ao sistema de saúde. Isso significa que deva ser identificada como o primeiro recurso de saúde a ser buscado pela comunidade quando há uma necessidade, servindo assim como referencial para o usuário. Para isso, se faz necessário o estabelecimento de um bom vínculo do usuário com a equipe de saúde bucal.

A longitudinalidade é outro princípio fundamental da APS cuja essência consiste na relação pessoal que se estabelece ao longo do tempo entre os indivíduos e o profissional ou uma equipe de saúde. Durante a residência o cirurgião-dentista tem a oportunidade de vivenciar essa relação com os pacientes, mesmo que essa experiência se limite a dois anos. A vivência torna-se ainda mais rica com o acompanhamento das relações dos pacientes com os profissionais há mais tempo na unidade de saúde e a observação dos registros do histórico dos pacientes nos prontuários, sendo essa evolução das informações de saúde dos usuários indispensáveis ao acompanhamento qualificado de cada membro e da família como um todo.

Um elemento importante usado para que os princípios da APS possam ser desenvolvidos é a territorialização. A unidade de saúde em que o residente atua trabalha com um território definido e uma população adscrita. O reconhecimento desse território é um passo básico para a caracterização da população e de seus problemas de saúde, bem como para a avaliação do impacto dos serviços sobre os seus níveis de saúde.

Para tal, fundamenta-se a formação para que o cirurgiãodentista egresso tenha subsídios para a compreensão da realidade local, numa prática orientada para o território e a família, considerando a diversidade e complexidade do contexto sóciohistórico-cultural, fundamental na compreensão dos sujeitos, ultrapassando o cuidado individualizado focado na doença, e contextualizando a saúde no espaço físico, social e relacional. Conhecer a família e a comunidade, seus membros, sua cultura, seus problemas de saúde, suas dificuldades e potencialidades, são subsídios fundamentais para o desenvolvimento de uma prática fundamentada na APS. Genograma, visitas domiciliares, perfil, diagnóstico e planejamento comunitário, estimativa rápida, atuação em conjunto com pontos de cultura, escolas, creches e associações comunitárias são algumas das ferramentas trabalhadas com os cirurgiões-dentistas no desenvolvimento desta competência.
A vigilância em saúde, muito além de uma ferramenta para o conhecimento dos indivíduos, família e comunidade, é um modelo de prática dentro desta nova perspectiva de orientação da saúde. Engloba os conceitos de vigilância epidemiológica, sanitária e ambiental, e de risco e vulnerabilidade em saúde (grupos, famílias, indivíduos) para o desenvolvimento de uma prática fundamentada no território. A vigilância, nessa perspectiva, tenta superar as discrepâncias entre as "práticas coletivas" de vigilância epidemiológica e sanitária e as "práticas individuais" da assistência, combinando estratégias de atenção, prevenção, promoção e educação em saúde com ênfase nos indivíduos e/ou grupos vulneráveis.

Ao encontro das estratégias de vigilância em saúde vem o modelo de atenção baseado em ações programáticas preconizadas na perspectiva de aumento da cobertura de atendimento a grupos de risco específicos ou baseados em linhas de cuidado e nos ciclos de vida, sob a ótica da promoção, prevenção e recuperação da saúde. Esta prática deve ser flexibilizada para adaptar-se às demandas cotidianas locais dos serviços de saúde, as quais os programas parecem não dar conta. No SSC do GHC destacam-se os programas da criança, gestantes, mulher, hipertensos e diabéticos, tuberculose, asma e HIVIAIDS. O egresso deve estar preparado para compreender, atuar, organizar e propor ações programáticas para o seu serviço, de saúde geral e/ou bucal, que passam a ser de responsabilidade e incorporadas a prática da equipe de saúde como um todo.

Além disso, pretende-se desenvolver a compreensão do cirurgião-dentista para a utilização/criação de indicadores de saúde no monitoramento das ações programáticas e acompanhamento das condições de saúde bucal das populações. Tais indicadores devem estar inseridos a um sistema de informações apropriado para organização, compilação e análise das situações de saúde dos usuários que possibilitem uma adequada avaliação e planejamento das ações.

Na formação também se agregam ferramentas para gestão e planejamento, através da vivência com o modelo de gestão colegiada nas unidades de saúde, durante 0 estágio de gerenciamento e nas pesquisas científicas na área aprimorando e qualificando a capacidade de análise crítica, de enfrentamento e proposição de ações dos profissionais (BRASIL, 2006).

\section{Considerações Finais}

A Residência Multiprofissional em Saúde pretende dirimir deficiências históricas de assistência e formação na área da Odontologia, capacitando o profissional para atuação na Atenção Primária a Saúde em consonância com os princípios e diretrizes do SUS. Além disso, preconiza-se a formação de cidadãos políticos, críticos e ativos, que busquem, em seus espaços de atuação, a construção coletiva de um projeto de saúde mais justo e qualificado, capaz de enfrentar os problemas que acometem os usuários, trabalhadores e o próprio sistema.

Tendo em vista a complexidade dessa formação em relação às pós-graduações convencionais, presenciais ou à distância, que muitas vezes não contemplam vivência prática em serviço, o profissional oriundo da RMS deve ser diferenciado no mercado de trabalho, dado o seu potencial de modificação do cenário da saúde bucal na APS e no contexto da ESF, tanto no nível da assistência como na gestão e pesquisa. Dessa forma, se faz necessário que os gestores reconheçam a valorizem esse profissional incorporando e pontuando a titulação nos editais de processos seletivos e concursos públicos da área. Cabe salientar ainda a necessidade de o Conselho Federal de Odontologia reconhecer adequadamente a Residência Multiprofissional em Saúde como uma formação diferenciada em

Rev. Fac. Odontol. Porto Alegre, v. 51, n. 3, p. 37-42, set./dez., 2010. 
nível de pós-graduação considerando as suas particularidades, em especial a alta carga horária e a formação na prática nos serviços de saúde.

\section{Referências}

BRASIL. Ministério da Saúde. Secretaria de Gestão do Trabalho e da Educação na Saúde. Departamento de Gestão da Educação na Saúde. Residência Multiprofissional em Saúde: experiências, avanços e desafios. Brasília: Ministério da Saúde, 2006.

BRASIL. Lei $n^{\circ}$. 11.129, de 30 de junho de 2005. Institui o Programa Nacional de Inclusão de Jovens - ProJovem; cria o Conselho Nacional da Juventude - CNJ e a Secretaria Nacional de Juventude; altera as Leis nos 10.683, de 28 de maio de 2003, e 10.429, de 24 de abril de 2002; e dá outras providências. Diário Oficial da União, Poder Executivo, Brasília, DF, 01 jul. 2005a.

BRASIL. Ministério da Saúde. Portaria n. ${ }^{\circ} 1.111$, de 05 de julho de 2005. Fixa normas para a implementação e a execução do programa de bolsa para a educação pelo trabalho. Diário Oficial da União, Poder Executivo, Brasília, DF, 07 jul. 2005b.

BRASIL. Ministério da Saúde; Ministério da Educação. Portaria n. ${ }^{\circ}$ 2.117, de 03 de novembro de 2005. Institui o programa de bolsas para Educação pelo Trabalho e cria a Comissão Nacional de Residência Multiprofissional em Saúde - CNRMS. Diário Oficial da União, Poder Executivo, Brasília, DF, 04 nov. 2005c.

BRASIL. Ministério da Saúde. Portaria Interministerial $n^{\circ} 45$, de 12 de janeiro de 2007. Dispõe sobre a Residência Multiprofissional em Saúde e a Residência em Área Profissional da Saúde e institui a Comissão Nacional de Residência Multiprofissional em Saúde. Diário Oficial da União, Poder Executivo, Brasília, DF, 15 jan. 2007.

BRASIL. Ministério da Saúde. Resolução $n^{\circ}$ 02, de 04 de maio de 2010. Dispõe sobre a organização, funcionamento e atribuições da Comissão de Residência Multiprofissional (COREMU) das instituições que oferecem programas de residência multiprofissional ou em área profissional da saúde. Diário Oficial da União, Poder Executivo, Brasília, DF, 05 mai. 2010a.

BRASIL. Grupo Hospitalar Conceição. Gerência de Ensino e Pesquisa. Cartilha da RIS/GHC. 2009a. Disponível em: <http://www2.ghc.com.br/GepNet/docsris/riscartilha2009.pdf>. Acesso em: 14 mar. 2010.

BRASIL. Grupo Hospitalar Conceição. Gerência de Ensino e Pesquisa. Projeto político pedagógico da RIS/GHC. 2009b. Disponível

<http://www2.ghc.com.br/GepNet/docsris/risprojeto.pdf>. Acesso em: 14 mar. 2010.

BRASIL. Grupo Hospitalar Conceição. Gerência de Ensino e Pesquisa. Estágio de gerenciamento - projeto 2010. 2010b. Disponível em:

<http://www2.ghc.com.br/GepNet/docsris/rismaterialdidatico15.pdf>.

Acesso em: 14 mar. 2010.

BRASIL. Ministério da Saúde. Secretaria de Atenção à Saúde. Departamento de Atenção Básica. Manual de especialidades em saúde bucal. Brasília: Ministério da Saúde, 2008.
MOYSÉS, S. T.; KRIGER, L.; MOYSÉS, S. J. Saúde bucal das famílias: trabalhando com evidências. São Paulo: Artes Médicas: 2008. Cap. 6, p. 125-135.

STARFIELD, B. Atenção Primária - Equilíbrio entre necessidade de saúde, serviços e tecnologia. Brasília: UNESCO, Ministério da Saúde, 2002. 726p. 